\title{
Physician Assistant Student Exposure to the Long-Term Care Setting by Working with a Consultant Pharmacist
}

\author{
Jill Morton*, Jessica Albers \& Vy Pham \\ Faculty: LaDonna S. Hale \\ Department of Physician Assistant
}

\begin{abstract}
This paper describes a unique, but replicable, opportunity for physician assistant (PA) students to obtain exposure to the long-term care (LTC) setting by shadowing a consultant pharmacist. Two students at a time spent four hours with the pharmacist, reviewing and co-presenting two to three patient charts. Students completed a questionnaire asking them to describe what they learned. The diseases and medication-related issues identified by students were consistent with those expected in LTC. Students indicated an increased appreciation of having strong knowledge of medication prescribing/monitoring, avoiding polypharmacy, disease prevention, the importance of multidisciplinary care, and preventing medication errors. Conclusion: This report describes a replicable educational opportunity providing PA students with exposure to the LTC setting through use of a consultant pharmacist.
\end{abstract}

\section{Introduction}

Physician assistant (PA) students must be trained to practice medicine as part of a multidisciplinary health care team in a wide range of settings including primary care and specialty practices across the lifespan. The Accreditation Review Commission on Education for the Physician Assistants (ARC-PA) requires programs to provide instruction that supports and develops competency in these six areas: 1) medical knowledge, 2) interpersonal and communication skills, 3) patient care, 4) professionalism, 5) practice-based learning and improvement, and 6) systems-based practice.[1] To effectively meet these six competency areas, PA programs must provide students with a variety of didactic and clinical educational experiences.

Exposure to the long-term care (LTC) setting can provide PA students with an excellent opportunity to gain skills in several of these key competency areas as well as help programs meet ARC-PA standards. Providing care to residents of LTC settings such as assisted living and nursing homes requires knowledge and management of acute, chronic, and preventative therapies and management of complex medical histories and complex medication regimens as well as a working knowledge of reimbursementrelated issues and multidisciplinary health care teams.
An early study found that adding a PA to a nursing home resulted in a $38 \%$ reduction in annual hospital admissions, a $69 \%$ reduction in total number of hospital days per 1000 patient years, and an increase in nursing home visits of 69\%.[2] In 2009, there were 18,160 practicing PAs in the U.S. and only $0.9 \%$ (84) reported their primary practice setting as LTC.[3] In order to improve healthcare in the LTC setting, increased geriatric-specific training is needed.

Although problematic to arrange and no longer specifically required by ARC-PA, LTC exposure is considered highly valuable and desirable. Early exposure to the geriatric population has been shown to increase interest in serving the geriatric population after graduation.[4] As the geriatric population continues to grow, training PAs in the LTC setting becomes increasingly critical.

Traditionally, physicians, PAs, and nurse practitioners serve as clinical supervisors to PA students. Anecdotally, programs have had a difficult time consistently providing clinical experiences in the LTC setting through these traditional clinical supervisors. Nursing homes and assisted living facilities are required by Federal and state law to have a consultant pharmacist regularly review patient charts and medication-related documentation, monitoring, and usage.[5] Thus, consultant pharmacists could serve as clinical supervisors for PA students in the LTC setting. A thorough review of the literature and PA educational websites was conducted. We were unable to find previous documentation of similar educational opportunities.

Study Purpose: Describe a unique, but replicable, clinical educational opportunity for PA students to obtain exposure to the LTC setting by shadowing a consultant pharmacist.

\section{Discussion and Significance}

LTC Setting: The LTC setting utilized was Carrington at Cherry Creek, a full-service assisted living facility in south Wichita, Kansas. The clinical supervisor was a consultant pharmacist and faculty member within the Wichita State University (WSU) 
PA program. During the first year of the WSU PA program, students spend most of their time in the classroom setting. Once weekly, students spend time observing assigned physicians, PAs, and advanced registered nurse practitioners throughout the community. The Cherry Creek facility was used as one of these observational opportunities. Two students at a time spent four hours on one occasion with the consultant pharmacist. Students reviewed each chart together, investigated their clinical questions using various medical references, identified medication irregularities, and then presented the patient orally to the pharmacist with their medicationrelated recommendations and discussion.

Descriptive Data Gathered: In order to describe the benefits of this educational opportunity from the students' perspective and to describe the types of patients, disease states, and medication issues seen by the students, students completed a questionnaire asking them to describe what they learned and to document the number of patient charts reviewed, patient characteristics, primary diagnosis/medical conditions, and types of medication problems identified. Students were also asked to describe: 1) the role of the consultant pharmacist in the LTC setting, 2) the importance of multidisciplinary collaboration in the LTC setting, 3) three medical issues common to older adults in the LTC setting, and 4) three things learned from the experience.

Questionnaire Responses: Of the 42 PA students in the program, 31 were assigned to attend the LTC rotation and 20 returned the questionnaire. Students documented a total of 49 charts reviewed ( 2 to 3 charts per student). The mean patient age was $76 \pm 13$ years; $73 \%$ were female; and $94 \%$ Caucasian. Thirty-eight different diagnoses were documented: mental illnesses $(n=33)$, hypertension $(n=30)$, gastrointestinal-related conditions $(n=23)$, coronary artery disease $(n=22)$, osteoporosis $(n=18)$, hyper/hypothyroidism $(n=13)$, heart failure $(n=13)$, and diabetes $(\mathrm{n}=12)$. The types of medication irregularities identified included: inadequate monitoring of labs/vitals (61\% of charts), missing documentation of medication purpose (47\%), excessive dosage (26\%), potentially serious drug interaction (18\%), and an indication for therapy without adequate treatment (14\%). Only $14 \%$ of charts had no medication irregularities.

The vast majority of the students described the role of the consultant pharmacist as providing medication review to enhance patient safety and improving medication management (appropriate purpose, dosing, avoiding interactions and contraindications, etc). Students described multidisciplinary collaboration in the LTC setting as important because the complexity of medical conditions requires expertise from many fields.

Discussion: This educational opportunity allowed students to observe the consultant pharmacist's role in the LTC setting. As expected, students were exposed to patients with a variety of disease states and medication-related issues. Students expressed an appreciation for the complexity of care and need for careful medication review and management. During the patient discussions, there were opportunities to assess and correct student deficits in medical knowledge, practice-based learning, and overall patient care skills; practice verbal patient presentation skills and professionalism; and to learn some aspects of systems-based practice related to prescribing, monitoring, and documentation regulations in the LTC setting. Students also witnessed firsthand multidisciplinary collaboration and team skills involved in the care of each patient.

\section{Conclusion}

This report describes a replicable educational opportunity providing PA students with exposure to the LTC setting through a consultant pharmacist. Gains in skills were not directly measured; however, students reported an increased appreciation for aspects of geriatric care that relate to all six Competencies for the PA Profession.

Manuscript has been submitted to Journal of Physician Assistant Education

\section{References}

[1] Competencies for the Physician Assistant Profession. National Commission on Certification of Physician Assistants. http://www.nccpa.net/PAC/Competencies home.aspx. Accessed July 18, 2010.

[2] Ackerman R, Kemle K. The effect of a physician assistant on the hospitalization of nursing home residents. JAGS. 1998;46(5):610-614.

[3] American Academy of Physician Assistants. National Physician Assistant Census Report. 2009 data (Table 3.4) http://www.aapa.org. Accessed July 18, 2001.

[4] Chen S, Melcher P, Witucki J, et al. Nursing home use for clinical rotations: taking a second look. Nursing and Health Sciences 2002;3:131-1137

[5] Kansas Administrative Regulations. Licensure of Adult Care Homes Regulations. 28-39-156: Pharmac 